\title{
Comparison of taste qualities elicited by tactile, electrical, and chemical stimulation of single human taste papillae
}

\author{
ARMAND V. CARDELLO \\ Food Sciences Laboratory \\ Behavioral Sciences Division, U.S. Army Natick Research \& Development Command \\ Natick, Massachusetts 01760
}

\begin{abstract}
Taste quality responses elicited by chemical, electrical, and tactile stimulation of 40 single fungiform papillae in four subjects were examined. A comparison of responses revealed differences in taste quality mediation for the three different classes of stimuli. Chemical stimulation elicited multiple taste qualities from a greater proportion of papillae than did either electrical or tactile stimulation. In addition, the chemical data revealed the presence of consistent bitter-sour and sour-salty confusions. Both tactile and electrical stimulation elicited relatively few bitter and sweet responses, as compared with either sour or salty responses. However, correct quality identification in these papillae for bitter and sweet compounds was no different from that for sour or salty compounds. In addition, electrical stimulation elicited a greater proportion of salty responses than did tactile stimulation. Comparison of the taste quality elicited by either tactile or electrical stimulation of a papilla with the taste quality exhibiting the greatest relative chemical sensitivity in the papilla also revealed independence of responding, and it was observed that both electrical and tactile stimulation elicited consistent taste quality responses from papillae in which these taste qualities could not be elicited by any concentration of any chemical test compound. The observed differences in quality judgments are discussed as possibly being the result of cross-modal gustatory associations resulting from nongustatory components of inadequate stimulation.
\end{abstract}

Several studies have examined taste quality mediation in response to chemical (Arvidson \& Friberg, 1980; Bealer \& Smith, 1975; Békésy, 1966; Cardello, 1978, 1979a, 1979b; Harper, Jay, \& Erickson, 1966; Kuznicki, 1978; McCutcheon \& Saunders, 1972), electrical (Békésy, 1964, 1965; Bujas, 1971; Bujas \& Mayer, 1977; Dzendolet \& Murphy, 1974; Plattig, 1972; Plattig \& Innitzer, 1976), and tactile (Bujas \& Mayer, 1977; Dzendolet \& Murphy, 1974; Plattig \& Innitzer, 1976) stimulation of single human taste papillae. However, few studies have compared the effects produced by these different stimuli on taste quality mediation in the same papillae. The only notable study of this kind was conducted by Békésy (1966), who reported a one-to-one correspondence in the taste qualities elicited from single fungiform papillae by chemical and electrical stimulation. Unfortunately, recent psychophysical studies have failed to corroborate Békésy's observations of quality specificity in these papillae, and have raised the likelihood that Békésy's chemical data were biased by his choice of stimulus concentrations and/or were due to nongustatory variables (Bealer \& Smith, 1975; Cardello, 1978; Harper et al., 1966; McCutcheon \& Saunders, 1972).

As a result of a recent study of the chemical sensitivities of 40 fungiform papillae in four subjects
(Cardello, 1978), the opportunity was provided to examine the responses of these same papillae to tactile and electrical stimulation. The present report summarizes the comparative results of these experiments and provides information relevant to (1) the confounding role of the tactile stimulus in studies involving electrical stimulation, (2) the effect of stimulus type on the number and nature of elicited taste qualities, (3) the dependence or independence of these qualities, and (4) the possible mechanism(s) underlying the sensory effects produced by inadequate stimulation.

\section{METHOD}

\section{Subjects}

The subjects were two males and two females, aged 18 to 25 . Each was screened for participation by the method reported in Meiselman and Dzendolet (1967) and each was requested not to eat, drink, or smoke within $1 \mathrm{~h}$ of each test session.

\section{Apparatus, Stimuli, and Procedure}

Chemical stimulation. The apparatus and procedure used for chemical stimulation have been reported elsewhere (Cardello, 1978). In brief, the apparatus consisted of a series of disposable, plastic 1-ml tuberculin syringes, fitted with 33-ga, blunt stainless steel hypodermic needles. Solution droplets $(.05 \mu \mathrm{l})$ were presented to the dorsal surface of each papilla with the aid of a binocular dissecting microscope (12X-60X). Nine test compounds were tested at room terperature $\left(25^{\circ} \mathrm{C}\right)$. The compounds and concentration ranges were: sucrose $(5-1,000 \mathrm{mM})$, dextrose $(2.5-1,500 \mathrm{mM})$, 
quinine sulfate (.01-3.00 mM), quinine monohydrochloride (.05$100 \mathrm{mM}), \mathrm{HCl}(.1-50 \mathrm{mM})$, citric acid (2.5-500 mM), NaCl (1$5,000 \mathrm{mM}), \mathrm{LiCl}(1-5,000 \mathrm{mM})$, and $\mathrm{KCl}(2.5-3,500 \mathrm{mM})$.

On each trial, the subject reported the taste quality of the solution. Response choices were "salty," "sweet," "sour," "bitter," "no taste," "indistinct or vague," and "complicated taste." The subjects were instructed to use the "indistinct or vague" category for weak and unidentifiable taste sensation and to use the "complicated taste" category for clear taste sensations that could not be classified as one of the four primary tastes. A 45-sec period, during which excess saliva was allowed to evaporate from the tongue surface, preceded each trial, and a 90 -sec interstimulus interval (ISI) was used. In addition, two control procedures were used to assess the extent of guessing. The first was to stimulate each papilla five times with distilled water. The second was to stimulate areas of the tongue where no papillae were present with solutions in the midrange of concentrations noted above. All control trials were interspersed with test trials and were undistinguishable from them in all respects.

A total of 69 papillae were prescreened with high concentrations of each compound in order to identify 10 papillae in each subject that were chemically responsive. All subsequent testing was conducted on only these chemically sensitive papillae.

Electrical and tactile stimulation. The apparatus for electrical stimulation consisted of an S4 stimulator (Grass Instrument Co.), adjusted to produce $.5-\mathrm{msec}$ monophasic, positive rectangular pulses. The voltage and frequency of stimulation were determined for each subject during preliminary trials, using a criterion that the elicited sensation be strong, but not so strong as to be disagreeable. Occasional variations in the voltage-frequency combination were made to assess the effect of these changes on perceived taste quality. The stimulating electrode was a length of fine-grade gold wire $(.3 \mathrm{~mm}$ diam) that was coated with insulating material ("insl- $X$ ") and that presented a stimulating surface of $.07 \mathrm{~mm}^{2}$. The return electrode was a disk-shaped silver । electrode $(8.0 \mathrm{~mm}$ diam), placed under the subject's tongue so that the congue could be extended in the same manner as during chemical stimulation. A $1 \mathrm{M} \Omega$ resistor was placed in series with the stimulating electrode to produce a constant current source and to protect the subject from current surges.

Tactile stimulation was effected using the smooth, blunt tip of the gold wire electrode described above. No current flowed through the electrode during trials involving only tactile stimulation.

Tactile and electrical stimulation were undertaken approximately 5 days after the completion of all chemical testing. As during chemical testing, trials involving tactile and electrical stimulation were preceded by a $45-\mathrm{sec}$ drying period. Following this period, the electrode was placed on the dorsal surface of a papilla and the subject was presented with $5 \mathrm{sec}$ of tactile stimulation. While accurate measurement of exerted pressure on the papilla was not feasible within the confines of this experiment, special effort was made to maintain constant pressure throughout all trials.

Following each "tactile trial," the subject was informed that a second trial would begin shortly. Within the next 5-10 sec, the current was presented for $5 \mathrm{sec}$. The stimulating electrode was then removed, and the subject rinsed his mouth with distilled water. Each of 10 papillae previously found to be chemically responsive in that subject was stimulated five times in random order, and a 2-min ISI was maintained.

Response choices during tactile and electrical stimulation were the same as during chemical stimulation and were made in the same manner. In addition, control trials, during which the stimulus was presented to areas of the tongue where no papillae were present, were again interspersed with test trials.

\section{RESULTS}

Taste qualities elicited on control trials during which test stimuli were presented to areas containing no taste papillae were random, totaling $6 \%$ for chem- ical stimulation, $5 \%$ for tactile stimulation, and $23 \%$ for electrical stimulation. Control trials during which distilled water was presented to the papillae resulted in "no taste" or "indistinct or vague" responses on $77 \%$ of the trials. Of the remaining responses, over $78 \%$ were "sour" or "bitter" responses reported by the two male subjects.

Of the 40 chemically sensitive papillae, $36(90 \%)$ were found to mediate gustatory sensations in response to electrical stimulation and $11(27 \%)$ were found to mediate gustatory sensations in response to tactile stimulation. These percentages can be compared to those of Plattig and Innitzer (1976), who found that $50 \%$ of fungiform papillae mediated taste sensations to anodal electric stimulation and $11 \%$ mediated taste sensations to tactile stimulation. The higher percentages observed here are most likely due to the fact that only papillae that were chemically responsive were tested, while in Plattig and Innitzer's study no chemical pre-screening was undertaken.

Table 1 shows the percentages of papillae that mediated one, two, three, and four taste qualities, as well as the specific combination of taste qualities elicited by each mode of stimulation. It is apparent that chemical stimulation produced a greater percentage of multiple taste qualities from these papillae than did either electrical or tactile stimulation. The possibility that this difference is attributable to the wide range of compounds and concentrations used during chemical testing, as compared with the limited number of stimulus parameters varied during tactile and electrical testing, is unlikely, since purposive changes in the frequency and voltage of the electrical stimulus and variations in the exerted pressure during tactile stimulation had no effect on the taste quality or qualities elicited from the papilla.

Table 2 (left panel) shows the percentage distribution of taste qualities elicited by tactile and electrical stimulation. Percentages are based on the total number of taste qualities elicited. The significance of differences in the proportions, both within and between modes of stimulation, were tested by comparison with

Table 1

Combinations of Taste Qualities Elicited From Individual Papillae by Chemical, Electrical, and Tactile Stimulation

\begin{tabular}{lccc}
\hline \multicolumn{1}{c}{$\begin{array}{c}\text { Gustatory } \\
\text { Quality }\end{array}$} & $\begin{array}{c}\text { Chemical } \\
(\mathrm{N}=40)\end{array}$ & $\begin{array}{c}\text { Electrical } \\
(\mathrm{N}=36)\end{array}$ & $\begin{array}{c}\text { Tactile } \\
(\mathrm{N}=11)\end{array}$ \\
\hline Salty-Sour-Sweet-Bitter & 80.0 & & \\
Salty-Sour-Bitter & 12.5 & 3.0 & \\
Salty-Sour-Sweet & 5.0 & 3.0 & \\
Sour-Bitter & & 3.0 & \\
Salty-Sour & & 11.0 & \\
Salty-Sweet & & 14.0 & 9.0 \\
Salty & 2.5 & 36.0 & 9.0 \\
Bitter & & 3.0 & 9.0 \\
Sweet & & 11.0 & 9.0 \\
Sour & & 28.0 & 64.0 \\
\hline
\end{tabular}

Note-Cell entries are the percentages of popillae mediating each combination of qualities. 
Table 2

\begin{tabular}{|c|c|c|c|c|}
\hline \multicolumn{3}{|c|}{$\begin{array}{l}\text { Percentage of Reported } \\
\text { Taste Quality }\end{array}$} & \multicolumn{2}{|c|}{$\begin{array}{l}\text { Percentage of Correct } \\
\text { Identifications }\end{array}$} \\
\hline & Tactile & Electrical & & Chemical \\
\hline Bitter & 5 & 2 & Bitter to Quinine Sulfate & 59 \\
\hline Sweet & 14 & 14 & Sweet to Sucrose & 98 \\
\hline Sour & 55 & 32 & Sour to Citric Acid & 63 \\
\hline Salty & 26 & 52 & Salty to $\mathrm{NaCl}$ & 62 \\
\hline
\end{tabular}

Note-The left panel shows the percentage distribution of taste qualities elicited by tactile and electrical stimulation of test papillae. The right panel shows the percent of correct quality identifications to presentation of $3.0 \mathrm{mM}$ quinine sulfate, $1,000 \mathrm{mM}$ sucrose, $500 \mathrm{mM}$ citric acid, and 5,000 $\mathrm{mM} \mathrm{NaCl}$ to the test papillae.

a chi-square distribution. For the tactile data, the proportions of bitter and sweet responses were both significantly smaller than the proportion of sour responses $(p<.05, \mathrm{df}=2)$. However, no differences were found among the proportions of bitter, sweet, and salty responses. For the electrical data, the proportion of sweet responses was significantly smaller than the proportion of salty responses $(\mathrm{p}<.05, \mathrm{df}=2)$ and the proportion of bitter responses was significantly smaller than those of every other taste quality $(p<.05, \mathrm{df}=2)$. A comparison of the tactile with the electrical data showed the proportion of salty responses to be significantly greater for electrical stimulation than for tactile stimulation $(\mathrm{p}<.05, \mathrm{df}=3)$. No other differences were significant.

In addition to the gustatory sensations noted above, tactile and electrical stimulation frequently elicited thermal "cooling" sensations and other sensations described as "metallic," "brassy," "tingling," "vibratory," or "buzzing" in all subjects.

The significance tests done on the data in the left panel of Table 2 suggest a relative lack of mediation of both bitter and sweet tastes to tactile and electrical stimulation of the test papillae. However, these results should not be interpreted as evidence of a qualityselective action of the inadequate stimuli on these papillae, unless evidence is provided that these papillae were, in fact, capable of mediating the bitter and sweet taste qualities to adequate (chemical) stimulation. At least two reasons exist as to why these papillae may not mediate these qualities to appropriate chemical stimuli. First is the fact that, through chance occurrence, this particular sample of 40 fungiform papillae may have fewer bitter and sweet receptor sites than do other fungiform papillae. Second, physiological and/or psychological taste "confusions" are known to occur, even under whole-mouth-stimulating conditions. The best identified example of this is the bitter-sour confusion, in which a sizable proportion of the population is known to describe normatively bitter compounds, such as quinine, as tasting sour and normatively sour compounds, such as acids, as tasting bitter (Gregson \& Baker, 1973; McAuliffe \& Meiselman, 1974; Meiselman \& Dzendolet, 1967; Robinson, 1970). Furthermore, this confusion is most readily observed near threshold (Gregson \& Baker, 1973). While the subjects used in the present experiment were screened to ensure a criterion level of quality identification using a whole-mouth technique, the relationship between perceived intensity and area of stimulation (Smith, 1971) has been shown to result in extremely high thresholds for single papillae (Cardello, 1978). As a result, sourbitter confusions that are not present during wholemouth stimulation may present themselves during single papilla stimulation. Further complicating quality identification in this situation are the reports of salty-sour confusions, both during stimulation of single fungiform papillae (Cardello, 1979b; Kuznicki, 1978; McCutcheon \& Saunders, 1972) and during stimulation of small areas of the anterior tongue (Cardello, 1979b; Collings, 1973; Nilsson, 1977).

The right panel of Table 2 shows the percentages of sweet, bitter, sour, and salty responses elicited by chemical stimulation of the test papillae with high concentrations of compounds characteristic of the four basic taste qualities. The solutions chosen for this test were $1,000 \mathrm{mM}$ sucrose, $3.0 \mathrm{mM}$ quinine sulfate, $500 \mathrm{mM}$ citric acid, and $5,000 \mathrm{mM} \mathrm{NaCl}$, respectively. While these data are conditionalized upon a quality response being given, as are the data in the left panel of Table 2, they are not presented for direct comparison with the tactile and electrical data, since they are not comparable measures. However, these data do show that this particular sample of papillae was capable of mediating bitter and sweet taste qualities to appropriate adequate stimuli and that the likelihood of correctly identifying these qualities was no worse (in the case of sweet, it was much better) than it was for the sour and salty qualities. Of as great an importance and interest, however, are the numbers and nature of the inappropriate quality responses to these compounds, since appreciable percentages of inappropriate responses were elicited by quinine sulfate, citric acid, and $\mathrm{NaCl}$.

Table 3 is a frequency distribution showing the specific nature of the inappropriate responses to these compounds for each subject. As reflected in the percentages in the right panel of Table 2, sucrose was appropriately identified as being sweet by all subjects. Quinine sulfate, on the other hand, was identified as both bitter and sour by three of the four subjects. Citric acid was identified as sour and salty by three subjects, and two subjects also identified it as bitter on some proportion of trials. $\mathrm{NaCl}$ was identified as both salty and sour by at least three of the four subjects.

The data presented in Tables 1 and 2 are based on taste quality responses across all 40 test papillae. 
Table 3

Frequency Distribution of Taste Quality Responses to 1,000 mM Sucrose, 3.0 mM Quinine Sulfate, $500 \mathrm{mM}$ Citric Acid, and $5,000 \mathrm{mM} \mathrm{NaCl}$ for Each Subject

\begin{tabular}{|c|c|c|c|c|c|c|c|c|c|c|c|c|c|c|c|c|}
\hline & \multicolumn{4}{|c|}{ Sucrose } & \multicolumn{4}{|c|}{ Quinine Sulfate } & \multicolumn{4}{|c|}{ Citric Acid } & \multicolumn{4}{|c|}{$\mathrm{NaCl}$} \\
\hline & Sweet & Bitter & Sour & Salty & Sweet & Bitter & Sour & Salty & Sweet & Bitter & Sour & Salty & Sweet & Bitter & Sour & Salty \\
\hline$S_{1}$ & 12 & 1 & 0 & 0 & 0 & 10 & 0 & 1 & 0 & 0 & 14 & 4 & 1 & 0 & 3 & 10 \\
\hline $\mathrm{S}_{2}$ & 10 & 0 & 0 & 0 & 1 & 7 & 3 & 0 & 0 & 0 & 5 & 10 & 3 & 0 & 2 & 5 \\
\hline$S_{3}^{2}$ & 13 & 0 & 0 & 0 & 0 & 4 & 8 & 2 & 0 & 2 & 12 & 3 & 0 & 0 & 5 & 10 \\
\hline $\mathrm{S}_{4}$ & 6 & 0 & 0 & 0 & 0 & 6 & 4 & 0 & 0 & 4 & 9 & 0 & 0 & 0 & 2 & 1 \\
\hline
\end{tabular}

Note-Frequencies are based on a total of 20 presentations of each compound to each subject. Null responses have not been included.

Taken together, they suggest differences both in the degree of specificity of quality responses from individual papillae and in the likelihood that certain qualities will be elicited by tactile, electrical, or chemical stimulation. However, these data do not provide direct information concerning the relationship between the specific chemical sensitivities of an individual papilla and the quality or qualities that are elicited from that papilla by tactile or electrical stimulation. Rephrasing this question, one may ask whether, when these papilla are grouped on the basis of their relative sensitivities to sweet, sour, bitter, and salty tasting substances, those papilla that are most sensitive to sweet (sour, etc.) compounds are also most likely to elicit a sweet (sour, etc.) taste to tactile or electrical stimuli. In order to address this question, all 40 papillae were categorized on the basis of their relative sensitivities to the various taste compounds. Sensitivity to each compound was assessed by determining recognition thresholds for each compound and each papilla. The threshold procedure was a modified method of constant stimuli that has been described in detail in a previous report (Cardello, 1978). In order to compare relative sensitivities among the papillae, recognition thresholds were converted to decibels, defined as $N(d B)=10 \log \left(E_{1} / E_{2}\right)$, where $N$ is the number of decibels, $\mathrm{E}_{1}$ is the threshold being converted to decibels, and $E_{2}$ is the average recognition threshold for that compound across all papillae. Papillae were then categorized on the basis of the characteristic quality of the compound showing the lowest relative recognition threshold (lowest $\mathrm{dB}$ level) in each papilla.

Tables 4 and 5 show the quality responses of these papillae, by category, to the tactile and electrical stimuli. Since $91 \%$ of all papillae responding to the tactile stimulus and $66 \%$ of all papillae responding to the electrical stimulus consistently mediated only a single quality, categorization of quality responding for these papillae was made on the basis of this quality. For those few papillae that mediated more than a single quality, categorization was made on the basis of the most frequently elicited quality.

The underlined entries on the diagonal in Table 4 constitute the number of papillae in which the taste quality elicited by tactile stimulation was the same
Table 4

Pairwise Categorization of Papillae According to the Taste Quality Most Frequently Elicited by Tactile Stimulation and the Taste Quality Associated With the Lowest Recognition Threshold Obtained by Chemical Stimulation

\begin{tabular}{ccccc}
\hline & \multicolumn{4}{c}{ Tactile Stimulation } \\
\cline { 2 - 4 } Stimulation & Sweet & Salty & Sour & Bitter \\
\hline Sweet & $\mathbf{0}$ & 0 & 1 & 0 \\
Salty & 0 & $\underline{0}$ & 3 & 0 \\
Sour & 1 & 0 & $\underline{3}$ & 0 \\
Bitter & 1 & 1 & $\mathbf{1}$ & $\underline{0}$ \\
\hline
\end{tabular}

Note-Cell entries are the number of papillae falling into each combination of taste quality categories. Entries are based on a total of 11 papillae that mediated taste qualities in response to both tactile and chemical stimulation.

Table 5

Pairwise Categorization of Papillae According to the Taste Quality Most Frequently Elicited by Electrical Stimulation and the Taste Quality Associated With the Lowest Recognition Threshold Obtained by Chemical Stimulation

\begin{tabular}{ccccc}
\hline & \multicolumn{4}{c}{ Electrical Stimulation } \\
\cline { 2 - 5 } $\begin{array}{c}\text { Chemical } \\
\text { Stimulation }\end{array}$ & Sweet & Salty & Sour & Bitter \\
\hline Sweet & $\frac{2}{3}$ & 0 & 1 & 0 \\
Salty & 1 & $\frac{6}{4}$ & 3 & 2 \\
Sour & 3 & 3 & $\frac{3}{5}$ & 0 \\
Bitter & 3 & 0
\end{tabular}

Note-Cell entries are the number of papillae falling into each combination of taste quality categories. Entries are based on a total of 36 papillae that mediated taste qualities in response to both electrical and chemical stimulation.

as the taste quality with the greatest relative chemical sensitivity in the papilla. A total of 3 papillae showed this correspondence. The likelihood of obtaining 3 or more such papillae, assuming independence between measures $[p($ match $)=.25]$, is .545 . The obtained proportion $(p=.273)$ of papillae with "matching" qualities has a .05 confidence interval of $.010<$ $\mathrm{p}<.536$, which includes the null hypothesis value $(\mathrm{p}=.25)$.

Table 5 shows a similar matrix for the correspondence between electrical and chemical stimulation. Of the 36 papillae mediating responses to both types of stimuli, 11 exhibited "matching" taste qualities. The likelihood of obtaining 11 or more papillae, 
assuming independence between the measures, is .295. The obtained proportion $(p=.306)$ has a .05 confidence interval of .156<p<.456 and again includes the null hypothesis value $(p=.25)$.

A further analysis of quality responding for these papillae revealed that $18 \%$ of the papillae tested with tactile stimulation consistently mediated taste qualities that could not be elicited from that papilla by any concentration of any of the nine chemical test compounds. Similarly, $8 \%$ of the papillae responding to electrical stimulation consistently mediated a taste quality that could not be elicited by any of the chemical test compounds.

\section{DISCUSSION}

Several procedural tests were undertaken in these experiments to insure the validity of the obtained data. First, all subjects were prescreened by a standardized procedure to insure that each knew and could correctly identify the sweet, salty, sour, and bitter qualities. Second, to insure that quality responses were not artificially restricted to the four "basic" taste qualities, a "complicated taste" category was included for alternative responses. However, this category was rarely used. Third, the results of the two control procedures indicated that these subjects were not guessing randomly. When the chemical or tactile stimuli were applied to papilla-free areas of the tongue, quality responses were minimal (5\%-6\%). The higher percentage of responses $(23 \%)$ for electrical stimulation may be due to spread of the current and can be assumed to be a maximal value. When distilled water was applied to the test papillae, taste quality responses were few in number and were nonrandom. These nonrandom responses were bitter and sour and are probably due to a natural "water taste" (Bartoshuk, 1968; Bartoshuk et al., 1964). Finally, a breakdown of quality responses to the chemical stimuli by individual subjects (Table 3 ) showed that all inappropriate quality responses could be attributed to the previously known quality confusions of sour-bitter and salty-sour.

The data in Table 1 show differences in the number of taste qualities elicited from individual papillae by the three modes of stimulation. As reported elsewhere (Cardello, 1978), the chemical data confirm earlier results of Bealer and Smith (1975), Harper et al. (1966), and McCutcheon and Saunders (1972), showing that single taste papillae mediate multiple taste qualities. However, the tactile and electrical data are characterized by a notable degree of quality specificity that was not found in the chemical data. These results provide some support for the earlier work of Békésy (1966) and Dzendolet and Murphy (1974), who found specificity in the responses to electrical stimulation of single fungiform papillae. However, $34 \%$ of the papillae responding to electrical stimulation in this experiment did, in fact, mediate multiple taste qualities. Furthermore, the lack of specificity in the chemical data is evidence that the tactile and electrical results are not related to any intrinsic specificity of the neural elements, but may be due to some selective action of the electrical and tactile stimuli on these elements and/or to nongustatory variables (see below).

Concerning the nature of elicited qualities, the data in Table 1 are consistent with Dzendolet and Murphy's (1974) report that the greatest number of papillae responding to electrical stimulation mediate the salty quality, especially if papillae mediating multiple taste qualities are taken into account. In addition, the predominance of sour responses to tactile stimulation gives some support to Bujas and Mayer's (1977) report that sour (and sweet) tastes dominate responding to tactile stimulation.

The data in the left panel of Table 2 show that bitter and sweet responses were elicited relatively infrequently by both tactile and electrical stimulation. Results of the significance tests on these data confirmed this. Yet, the data in the right panel of Table 2 show that both of these qualities could easily be elicited by adequate stimulation of the same papillae and that quality identification was no worse than for the sour or salty qualities. Together, these data suggest a quality-selective action of nonadequate stimulation. In the case of electrical stimulation, this selective action could result from hydrolysis of saliva. However, a nonpolarizable gold electrode and a short pulse duration were used in these experiments, and both of these factors would minimize hydrolysis effects. Second, the predominant quality response to electrical stimulation was salty, not sour, as would be expected if hydrolysis was occurring. (Note, however, that some proportion of salty responses might be expected upon hydrolysis, due to the sour-salty confusion noted previously.) Since tactile stimulation produced predominantly sour responses, which cannot be explained by hydrolysis, and a significantly greater proportion of salty responses was elicited by electrical stimulation than by tactile stimulation, it appears that the actual effect of the electrical current was to elicit a salty taste that either inhibited or masked the sour taste produced by the tactile stimulus.

The data in Tables 4 and 5 indicate that the taste qualities elicited by tactile or electrical stimulation of fungiform papillae are independent of the quality associated with the chemical compound having the lowest relative recognition threshold in the papilla. Of equal importance to the question of independence of quality judgments is the fact that $8 \%$ of the papillae that responded to electrical stimulation and $18 \%$ of the papillae that responded to tactile stimulation consistently mediated taste qualities that could not be elicited from these papillae by any concentration of the nine chemical test compounds. Any complete explanation of the gustatory effects produced by in- 
adequate stimulation must account for this peculiar independence in taste quality mediation between adequate and inadequate sources of stimulation.

The observed differences in the examined aspects of taste quality responding to chemical, electrical, and tactile stimulation of single fungiform taste papillae raise questions concerning the mode of action of inadequate stimuli. As stated previously, both the procedures and data obtained in these experiments argue against an explanation of the electrical data on the basis of hydrolysis. Previous demonstrations of the independence of electrical and chemical stimulation, such as the failure of potassium gymnemate to block cathodal sweet and bitter tastes (Bujas \& Pfaffmann, 1971) and the failure of chemical adaptation to affect responses to electrical stimulation (Bujas et al., 1974), have been interpreted as being the result of the electrical stimulus's bypassing the receptor mechanisms of taste and producing direct stimulation of first-order neural elements. Such direct stimulation of first-order elements may well account for much of the independence between the chemical and electrical data. However, the similar quality independence between the chemical and tactile data is less easy to explain.

In view of the differences in quality responding between adequate and nonadequate stimulation, an alternative or complementary explanation is proposed here to explain the consistency of quality responses to both tactile and electrical stimulation of single papillae. This explanation is based on cross-modal associations resulting from nongustatory components of tactile and electrical stimulation. Marks (1978) has presented a summary of evidence for the occurrence of cross-modal quality associations among sensory systems, including those between taste and the other senses. In the results section, it was pointed out that both tactile and electrical stimulation produced discrete tactile and thermal sensations in all subjects. Based on the introspective reports of these subjects, it appears likely that these nongustatory sensations were often interpreted as gustatory qualities. One subject likened the task to that of verbal association, in which "you show me the color green and I say it's peppermint," another subject said she could "relate the sensations (of electrical stimulation) to the sensations of chemicals, but the taste was not the same," and a third said, "The tingling of this salty taste (electrical) is like the tingle of chemical salts at high concentrations." Similar references to cross-modal associations were frequent among subjects and may be responsible for many of the consistent quality reports given in response to the inadequate stimuli.

The development of associations between nongustatory components of tactile and electrical stimulation and specific taste qualities is reasonable, especially if one considers that "stinging," "biting," and similar tactile sensations frequently accompany the sour and salty tastes of acids and salts (Abrahams, Krakauer, \& Dallenbach, 1937; Holway \& Hurvich, 1937; Moncrieff, 1967; Hunt, Dubose, \& Meiselman, Note 1). The fact that the majority of responses to tactile and electrical stimulation in this study, as well as in others, have been sour or salty suggests that the reverse of this association may be responsible for many of the obtained taste quality responses to these stimuli.

The failure to observe correspondence in the taste qualities elicited by electrical and chemical stimulation of the same papilla contradicts Békésy's (1966) earlier comparative work. The similar lack of correspondence between tactile and chemical stimulation suggests that the sensitivity of a papilla to either form of inadequate stimulation is independent of its chemical sensitivities. Such independence may be due to a differential sensitivity of the neural gustatory elements to these classes of stimuli or, as suggested here, to cross-modal associations resulting from nongustatory components of tactile and electrical stimulation. Further research is needed on the comparative effects of both adequate and inadequate stimulation of the taste system, as well as on the separation of gustatory from nongustatory effects in studies involving inadequate stimulation.

\section{REFERENCE NOTE}

1. Hunt, D., Dubose, C., \& Meiselman, H. Individual subject consistency and tactile stimulus component as factors in taste adaptation experiments. Paper presented at the Annual Meeting of the Eastern Psychological Association, Boston, 1977.

\section{REFERENCES}

Abrahams, H., Krakauer, D., \& Dallenbach, K. Gustatory adaptation to salt. American Journal of Psychology, 1937, 49, 462-469.

Arvidson, K., \& Friberg, V. Human taste: Response and taste bud number in fungiform papillae. Science, 1980, 209, 807-808.

Bartoshuk, L. M. Water taste in man. Perception \& Psychophysics, 1968, 3, 69-72.

Bartoshuk, L. M., McBurney, D., \& Pfaffmann, C. Taste of sodium chloride solution after adaptation to sodium chloride: Implications for the "water taste." Science, 1964, 143, 967-968.

Benler, S. L., \& Smith, D. V. Multiple sensitivity to chemical stimuli in single human taste papillae. Physiology \& Behavior, 1975, 14, 795-799.

BÉkÉsY, G. von. Sweetness produced electrically on the tongue and its relation to taste theories. Journal of Applied Physiology, $1964,19,1105-1113$.

BÉ KÉSY, G. von. Temperature coefficients of the electrical thresholds of taste sensations. Journal of General Psychology, 1965, 49, 27-35.

BÉKÉSY, G. von. Taste theories and the chemical stimulation of single papillae. Journal of Applied Physiology, 1966, 21, 1-9.

BujAs, Z. Electrical taste. In L. M. Beidler (Ed.), Handbook of sensory physiology (Vol. 4) Chemical senses. 2: Taste. New York: Springer-Verlag, 1971.

BuJAs, Z., \& MAYER, D. Sensory effects of continuous and 
iterative electrical stimulation of the tongue. Acta Instituti Psychologici Universitatis Zagrabiensis, 1977, 79, 3-16.

Bujas, Z., \& Pfaffmann, C. Potassium gymnemate and the sweet and bitter taste evoked electrically. Perception \& Psychophysics, 1971, 10, 28-29.

Bujas, Z., Szebo, S., Kovacic, M., \& Rohace K, A. Adaptation effects on evoked electrical taste. Perception \& Psychophysics, $1974,15,210-214$.

Cardello, A. V. Chemical stimulation of single human fungiform taste papillae: Sensitivity profiles and locus of stimulation. Sensory Processes, 1978, 2, 173-190.

Cardello, A. V. Psychophysical exponents for single papillae: A comparison with whole-mouth exponents. Perception \& Psychophysics, 1979, 25, 510-516. (a)

Cardello, A. V. Taste quality changes as a function of salt concentration in single human taste papillae. Chemical Senses \& Flavour, 1979, 4, 1-13. (b)

Collings, V. B. Human taste response as a function of locus of stimulation on the tongue and soft palate: Implications for sensory coding. Unpublished doctoral dissertation, University of Pittsburgh, 1973.

Dzendolet, E., \& Murphy, C. Electrical stimulation of human fungiform papillae. Chemical Senses \& Flavour, 1974, 1, 9-15.

Gregson, R. A. M., \& BAKER, A. F. H. Sourness and bitterness: Confusions over sequences of taste judgements. British Journal of Psychology, 1973, 64, 71-76.

Harper, H., JAY, J., \& Erickson, R. Chemically evoked sensations from single human taste papillae. Physiology \& Behavior, 1966, 1, 319-325.

Holway, A., \& Hurvich, L. Differential gustatory sensitivity to salt. American Journal of Psychology, 1937, 49, 37-48.

KUZnICKI, J. Taste profiles from single human taste papillae. Perceptual and Motor Skills, 1978, 47, 279-286.
MARKs, L. E. The unity of the senses: Interrelations among the modalities. New York: Academic Press, 1978.

McAuliffe, W. K., \& Meiselman, H. L. The roles of practice and correction in the categorization of sour and bitter taste qualities. Perception \& Psychophysics, 1974, 16, 242-244.

McCutcheon, N. B., \& Saunders, J. Human taste papilla stimulation: Stability of quality judgments over time. Science, $1973,175,214-216$.

Meiselman, H. L., \& Dzendolet, E. Variability in gustatory quality identification. Perception \& Psychophysics, 1967, 2 , 496-498.

MonCriefF, R. W. The chemical senses. Cleveland, Ohio: CRC Press, 1967.

Nilsson, B. Taste activity of the human palate. In: Studies with electrogustometry and taste solutions on young adults. Acta Odontologica Scandinavica, 1977, 35, 51-62.

Plattig, K. On the specificity of single human tongue papillae studied by electric stimulation. In D. Schneider (Ed.), Olfaction and taste (Vol. 4). Stuttgart: Wissenschaftliche Verlagsgeschaft MBH, 1972.

Plattig, K., \& Innitzer, J. Taste qualities elicited by electric stimulation of single human tongue papillae. Pflügers Archiv, 1976, 361, 115-120.

RoB Inson, J. O. The misuse of taste names by untrained observers. British Journal of Psychology, 1970, 61, 375-378.

Sмгтн, D. V. Taste intensity as a function of area and concentration: Differentiation between compounds. Journal of Experimental Psychology, 1971, 87, 163-171.

(Received for publication May 8, 1980; revision accepted November 7, 1980.) 